Running Head: REVIEWING THE STRUCTURE OF MOTIVATION

\title{
Putting the Pieces Together: Reviewing the Structural Conceptualization of Motivation within SDT
}

\author{
Joshua L. Howard ${ }^{1}$, Marylene Gagné2,3, \& Alexandre J. S. Morin ${ }^{4}$ \\ ${ }^{1}$ Department of Management, Monash University, Melbourne, Australia. \\ ${ }^{2}$ Future of Work Institute, Curtin Business School, Perth, Australia \\ ${ }^{3}$ University of Western Australia \\ ${ }^{4}$ Substantive-Methodological Synergy Research Laboratory, Department of Psychology, Concordia \\ University
}

Corresponding author: Joshua L. Howard, Monash Business School, Department of Management, Monash University, 900 Dandenong Rd, Caulfield East VIC 3145, Australia.

Email: josh.howard@monash.edu

This is the prepublication version of the following manuscript:

Howard, J.L., Gagné, M., Morin, A.J.S. (2020). Putting the Pieces Together: Reviewing the Structural Conceptualization of Motivation within SDT. Motivation \& Emotion. Early view:

https://doi.org/10.1007/s11031-020-09838-2

\begin{abstract}
Self-determination theory presents a multi-dimensional approach to human motivation in which motivation is driven by a range of classifiably distinct regulation styles. However, these different regulations are also considered to fall along a continuum of self-determination which implies that a global dimension reflecting the degree of self-determination present is important. The lack of explicit integration between these two aspects in the conceptualization of motivation has recently led to debates and a flux of research on the structure of motivation as defined in self-determination theory. This review comprehensively explores recent advances in this area as well as more classical approaches in order to establish an optimal and empirically supported conceptualization of motivation. A novel solution is proposed, namely a semi-radex structure of motivation, in which types of motivation are predictably ordered by degree of self-determination while also maintaining their unique contribution as distinct factors. Theoretical and practical implications for researchers who use selfdetermination theory are made.
\end{abstract}

Keywords: self-determination theory; motivation; continuum; semi-radex; bifactor exploratory structural equation modeling (B-ESEM). 


\section{Putting the Pieces Together: \\ Reviewing the Structural Conceptualization of Motivation within SDT}

A common and recurrent theme within motivation research concerns the number of factors required to appropriately and completely capture an individual's motivation. McClelland (1987) depicted motives in terms of the needs for achievement, power, and affiliation, whereas others identified approach and avoidance mindsets as the key defining characteristics of motivation (e.g., Higgins, 1997). Additional theories rely on a broad definition of motivation, and rather focus on the mechanisms that underpin motivated behavior (e.g. Expectancy Theory, Theory of Planned Behavior, and Goal-setting Theory; Locke \& Latham, 1990; Fishbein \& Ajzen, 1977; Vroom, 1964). SelfDetermination Theory (SDT; Deci \& Ryan, 1985; Ryan \& Deci, 2017) is more closely aligned with the former of these approaches and specifies several motivational regulations that energize and guide individual behavior based on how self-determined or volitional each regulation is. According to SDT, each type of regulation leads to different outcomes in terms of wellbeing, performance, persistence and commitment to a course of action (Cerasoli, Nicklin, \& Ford, 2014; Gagné et al., 2015; Ng, Ntoumanis, Thogersen-Ntoumani, Deci, Ryan, Duda, \& Williams, 2012). However, SDT also assumes that these regulations fall along a single underlying continuum of according to their degree of selfdetermination (also referred to as relative autonomy).

Even though SDT explicitly notes the importance of recognizing the conceptually distinct nature of these various types of behavioral regulations (i.e., a multidimensional approach), the continuum hypothesis might be erroneously taken to suggest that the regulations themselves do not really represent distinct factors, but merely points along a single continuum of self-determination (i.e., a onedimensional approach). This simplified one-dimensional interpretation could in turn suggest that human motivation can be measured through a single measure or index of self-determined motivation (such as the relative autonomy index, or RAI, proposed by Grolnick \& Ryan, 1989). These operationalizations do not clearly align with SDTs multidimensional conceptualization of motivation as encompassing a series of qualitatively distinct types of behavioral regulations, thereby raising a variety of questions concerning how best to conceptualize and assess human motivation. Is motivation best conceptualized and measured as a single entity characterized only by the degree of selfdetermined motivation? Is it necessary to consider the unique characteristics of the different motivation types? In this article, we argue that it is necessary to consider both simultaneously. Indeed, although both components of SDTs conceptualization of human motivation (e.g., the one-dimensional continuum hypothesis and the multidimensional nature of behavioral regulations) caries valuable information, they both provide an incomplete picture of human motivation when considered on their own. However, the assumption that both components are required to achieve a complete understanding of human motivation raises yet another question on how best to integrate these components as part of a single theoretical and analytical framework. It is important to address these questions as "[T]he surest way to make progress in science is to improve on the conceptual definition of what one is studying" (Reeve, 2016, p. 31). If we do not clarify questions around the compatibility and integration of the multidimensional conceptualization of human motivation and of the one-dimensional continuum structure of motivation, researchers are left with a disparate array of measurement methods that can lead to a body of research results that is very hard to integrate in any consistent manner. This, in turn, may drastically slow progress in developing tools and interventions to improve motivation across different life domains.

This review paper synthesizes the piecemeal research conducted on this topic to date and clarifies the distinction between the continuum of self-determination and the categorical types of motivation within SDT and how they relate to one another. We integrate evidence from a wide range of analytical approaches including basic examination of correlational structures, multidimensional scaling, factor analysis, and profile approaches, and suggest a refined conceptualization of self-determined 
motivation - a semi-radex structure. Finally, we discuss the theoretical and practical implications of this conceptualization and the benefits it may hold for future SDT research.

\section{Behavioral Regulations According to Self-Determination Theory}

SDT defines behavioral regulations as the reasons why activities are pursued, proposing six types of regulations, depicted in Figure 1. These types of regulation vary in respect to a perceived locus of causality (deCharms, 1968; Ryan \& Connell, 1989), in which types of motivation at one end are related to a greater sense of autonomy or agency over one's own actions, and types at the other end relate to a sense of being controlled or lacking autonomy. This concept of perceived locus of causality is also referred to in SDT as a continuum of autonomy or self-causality (Ryan \& Connell, 1989, and as a continuum of self-determination by Howard, Gagné, \& Bureau, 2017). Each of these terms indicate a predictable ordering of motivation types based upon an underlying concept of experienced autonomy and a sense of being the origin of one's own behavior (deCharms, 1968).

Intrinsic motivation describes purely self-determined motivation and characterizes the highest level of perceived locus of causality. An individual driven by purely intrinsic motives enjoys the behavior or finds it interesting and pleasant in and of itself. Intrinsic motivation does not require external reinforcement and may be diminished by it (Deci, Koestner, \& Ryan, 1999; 2001). Promoting and maintaining intrinsic motivation should lead to optimal functioning, defined as a "manifestation of intra- and interpersonal growth and development in terms of employee well-being (e.g., positive emotions, vitality), attitudes (e.g., job satisfaction, organizational commitment), and behavior (e.g., performance, proactivity, and collaborative behaviors" (Van den Broeck, Carpini, \& Dieffendorf, 2019 , p. 30). The remaining regulations are classified as extrinsic forms of motivation that vary in the extent to which they are internalized and therefore self-determined. Internalization, defined as an "active assimilation of behavioral regulations that are originally alien or external to the self" (Ryan, 1995, p. 405), is what can transform extrinsic motivation into something closer, yet different from, intrinsic motivation.

Integrated regulation, the most highly internalized type of external regulation, involves deep internalization of values into an individual's sense of self. As such, individual pursuing behavior for this motive will experience this behavior as an integral part of their identity and a clear reflection of their sense of self. This type of motivation, while theorized about, is rarely measured due to substantial overlap with neighboring motives. In addition to scale validation studies failing to identify it as a distinguishable type of motivation (e.g. Gagne et al., 2015; Vallerand et al., 1992), recent a metaanalytic study examining the structure of motivation using multidimensional scaling also concluded that it is empirically indistinguishable (Howard et al., 2017).

Identified regulation is the next most highly internalized form of extrinsic motivation and involves the recognition of the inherent instrumental value of the behavior. Individuals experiencing identified regulation find the behavior to be meaningful and important, hence they willingly and actively pursue it, without reliance on coercive external or internal forces. Identified regulation is an autonomous form of motivation, while remaining distinct from intrinsic motivation. Whereas intrinsic motivation is related to behaviors that are valued in and of themselves, identified regulation is related to behaviors that are expected to lead to valued outcomes. Identified regulation has proven to be a strong predictor of variables such as vitality and positive affect ( $\mathrm{Ng}$ et al., 2012), and has sometimes been shown to be an even more effective predictor of positive results than intrinsic motivation (Losier \& Koestner, 1999; Ng et al., 2012). Behavior driven by identified regulation is likely to be maintained over a longer time frame than less autonomous regulations, given the more internalized nature of the rewards being pursued (Deci, Koestner, \& Ryan, 1999; 2001).

Introjected regulation refers to behaviors driven by the avoidance of a negative self-image and self-focused emotions (e.g., guilt, shame), or by the experience of a positive self-image and selffocused emotions (e.g., pride). Introjected regulation is described as self-applying rewards and 
punishments to oneself (i.e., internal pressure). As such, introjected regulation is conceptualized as a partially internalized type of extrinsic motivation (Deci \& Ryan, 2000), or a type of motivation with a moderate level of perceived locus of causality. This type of regulation remains dependent on contingent consequences, albeit self-administered ones, and therefore is unlikely to be maintained when dissociated from the outcome (Koestner, Losier, Vallerand, \& Carducci, 1996). This dual nature of introjected regulation is demonstrated by meta-analytic findings of relations between introjection and a variety of negative (e.g., depression, anxiety) and positive (e.g., engagement in physical education, maintaining a healthy diet) outcomes (Ng et al., 2012).

External regulation is the least internalized form of extrinsic regulation, with the most externally focused locus of causality, and refers to behaviors that are coerced by the promise (or threat) of external rewards (or punishments). Rewards and punishments can take tangible (e.g., bonuses, getting fired) or social forms (e.g., approval, criticism). Individuals acting under this type of regulation do not find the behavior interesting or enjoyable but seek to attain (or avoid) the associated rewards (or punishments). Even though this type of regulation does lead to the target behavior, research evidence suggests that it is likely to lead to short-term and lower quality engagement in the behavior (Deci, Koestner, \& Ryan, 1999), and to have a detrimental impact on wellbeing (Deci \& Ryan, 2000; Gagné \& Deci. 2005). For instance, a meta-analysis conducted by Ng and colleagues (2012) showed that while external regulation was unrelated to physical activity and health outcomes, it was positively associated with depression, anxiety, and negative affect, and negatively related to vitality and quality of life. Similarly, Cerasoli, Nicklin, and Ford (2014) found that while both external incentives and intrinsic motivation contributed to performance quantity, only intrinsic motivation was related to performance quality.

Lastly, amotivation is defined as a complete lack of intention to enact the target behavior, as opposed to holding any intrinsic or extrinsic forms of motivation (Deci \& Ryan, 2000). Predictably, amotivation is uniformly associated with poor performance (e.g., proactivity, effort, accomplishments; Gagné et al., 2015) and wellbeing (e.g., depression, anxiety, affect, quality of life; $\mathrm{Ng}$ et al., 2012). While the role of amotivation within motivation research is somewhat ambiguous (Chatzisarantis, Hagger, Biddle, Smith, \& Wang, 2003), we include it where appropriate.

\section{The Current Structure of Motivation in SDT}

SDT emphasizes that these regulation types are qualitatively distinct from one another and serve different functions in terms of prediction (mediation, moderation, etc.), but are also tied together in a predictable fashion along a single overarching continuum of self-determination. While this model (See Figure 1) has given rise to a wealth of well-conducted and insightful research, it also fails to integrate its various facets or clearly specify how the various regulations can be both separate constructs and yet points along a continuum. As such, research hypotheses to date typically refer to either the regulations as separate constructs or to the general level of self-determination, but rarely to both, thereby systematically ignoring one half of the theory. This leads to a range of difficulties including appropriate hypothesis development, comparisons between studies utilizing different approaches, and in many instances, difficulties (or impossibilities) in disentangling which of these elements (the unique motivational quality or the global degree of self-determination) is crucial to observed effects. For example, a study examining the full range of regulations in predicting well-being may find a positive association between identified regulation and the outcome, however it is not possible to distinguish if this result is due to a unique characteristic of identified regulation (i.e., meaningfulness) or to the global degree of self-determination. Alternatively, studies relying on a single score reflecting participants global levels of self-determination (such as the RAI) will be able to examine how the degree of self-determination associates with covariates but will be unable to consider the unique contributions of each regulation subscale (i.e., thereby treating motivation as a single-dimensional construct). This exemplifies a major pitfall of the current conceptualization of motivation as the lack 
of clarity and integration of these two aspects (degree of self-determination and specific regulation types) allows for divergent assumptions which come with theoretical and practical implications that are much too strong to be left unchecked.

The currently accepted conceptualization of motivation and the theory surrounding it raises two unanswered questions. Firstly, how can we theoretically integrate into a clear conceptualization qualitatively distinct motivational concepts ordered along a single continuum? Secondly, how can we operationalize this integrative conceptualization in empirical research? Refining self-determination theory to explicitly acknowledge these motivational characteristics is necessary for the advancement of research in the field of motivation (Reeve, 2016).

Therefore, the objective of this review is to examine all the available evidence concerning the structural conceptualization of motivation offered by SDT and refine this conceptualization in order to guide future research more clearly. The following sections present an exhaustive list of the methods previously used to inform this structural issue. Results from these diverse approaches are then compared and integrated before a refined conceptualization of SDT motivation proposed - a semiradex structure of human motivation. Finally, theoretical and practical implications are discussed.

\section{Research Evidence for the Continuum Hypothesis of Motivation and the Unique Quality of Each Behavioral Regulation}

\section{Correlation Matrices}

The first approach used to verify the SDT continuum structure has been to examine whether the pattern of correlations obtained among scores on various forms of behavioral regulations would follow a simplex structure. This simplex pattern would require higher correlations between more theoretically proximal regulations and weaker correlations between more distal regulations. To cite the authors who introduced the simplex approach to SDT:

"The simplex concept is derived from Guttman's (1954) radex theory, which describes ordered relations between correlated variables. In a simplex, variables are ordered in terms of complexity or conceptual similarity, such that those deemed more similar correlate more highly than those that are hypothetically more discrepant" (Ryan \& Connell, 1989, p. 750).

In a correlation matrix in which the behavioral regulations are organized a priori based on their expected position on the continuum, the highest correlations should be observed along the diagonal with a tapering effect such that correlations become lower the further they are from the diagonal. In essence this pattern tests whether the regulations are in the correct order relative to each other.

Additionally, this simplex structure has been used to support one-dimensional composite scores of global motivation such as the RAI (Grolnick \& Ryan, 1989), which is created through the weighted aggregation of regulation subscales according to their hypothesized position on the continuum. The vast majority of these ad-hoc and eye-ball analyses of a simplex pattern of correlations have confirmed the predictable ordering of regulation types.

In an attempt to more systematically assess the adequacy this simplex representation, Ryan and Connell (1989) proposed an "adjacency index" to mathematically summarize the pattern of intercorrelations between the regulations. For example, the correlation between external and introjected regulations is designated by a value of 3 , the one between external and identified regulations is designated by a value of 2 , and the one between external regulation and intrinsic motivation is designated by a value of 1 . These weights are then used in regressions to predict the squared correlations obtained among behavioral regulation scores. In this study, the authors found support for a simplex structure. Despite its appeal as an initial attempt to systematize what had been, so far, unsystematic tests of the continuum structure of motivation, this approach has since been criticized as being insensitive to clear divergences from a perfect simplex structure (Chemolli \& Gagné, 2014; Fernet, Senécal, Guay, Marsh, \& Dowson, 2008; Guay, Vallerand, \& Blanchard, 2000).

More recently, a large-scale meta-analysis has examined this proposed simplex-like structure 
across five major domains and 486 independent samples, finding that the simplex structure provided an accurate representation of the vast majority of SDT research (Howard et al., 2017). However, even after moderation analyses were performed, heterogeneity remained unaccounted for in the relations between the types of behavioral regulations. This heterogeneity indicates that, while the simplex pattern was reproducible, the exact distances between regulations varies, suggesting the presence of meaningful specificities at the subscale level.

A final and related examination of relations between regulation factors has been conducted using path analysis and structural equation modelling. This approach consists of creating a path model in which paths are only permitted between adjacent regulation factors (defined from their items) while other paths are constrained to be zero (i.e., amotivation -> external -> introjected -> identified -> intrinsic). Results of a study conducted by Li and Harmer (1996) in the sport motivation area based on this approach largely supported the presence of a simplex structure, and the idea that most relations involving non-adjacent factors were indirect and mediated by the a priori simplex pattern. However, their results also revealed the need to include smaller direct effects among non-adjacent factors (external regulation and intrinsic motivation in males, and amotivation and intrinsic motivation in females), suggesting that the relations may not be entirely captured by linear dependencies among adjacent factors. Additional studies in the health domain replicated these results but disagreed on the need for additional paths (Li, 1999) or on the exact nature on these additional paths (Mallett, Kawabata, Newcombe, Otero-Forero, \& Jackson, 2007). In an attempt to synthesize these results, Chatzisarantis and colleagues (2003) conducted a meta-analysis of 21 published articles in which the perceived locus of causality scale (Ryan \& Connell, 1989) was used to measure motivation. The study used the same path analytic procedure described above relying on correlation matrices corrected for measurement and sampling error and generally found support for a simplex pattern of correlations ranging from external regulation to introjected and identified regulation. However, they concluded that the extreme ends of the continuum (i.e., intrinsic motivation and amotivation) did not follow the expected simplex pattern, thus suggesting a potentially more nuanced conceptualization of the SDT continuum. Taken together, these tests of a simplex-like structure have largely provided support for a one-dimensional continuum of self-determination.

\section{Factor Analysis}

The multidimensional factor structure of SDT-based motivation measures have been extensively verified using a combination of exploratory factor analyses (EFA) and confirmatory factor analyses (CFA). Verifying the adequacy of these underlying measurement models, estimated at the item level, is important as they represent a statistically testable representation of motivation. These studies have almost invariably found support for SDT's multidimensional representation of human motivation as encompassing a variety of distinct types of behavioral regulation, with more than 10 validated measures of motivation adopting a multidimensional solution (e.g., Gagné et al., 2015; Li, 1999; Lonsdale, Hodge, \& Rose, 2008; Pelletier et al., 1995; Pelletier, Rocchi, Vallerand, Deci, \& Ryan, 2013; Ryan \& Connell, 1989; Vallerand et al., 1992).

Some authors have specifically relied on factor analyses to test the continuum hypothesis by contrasting the relative efficacy of the a priori multidimensional factor structure of these instruments with simpler one- (global self-determination) and two- (autonomous and controlled motivation) factor structures. These studies have consistently rejected the simplified one-factor solution in favor of the a priori multidimensional structure (Gagné et al., 2015; Li, 1999; Lonsdale et al., 2008). Although these results are informative, it is worth noting that SDT does not suggest that the various behavioral regulations should only form a single underlying dimension, but rather that they are conceptually distinct from one another in addition to being organized along a continuum.

More advanced methods, such as exploratory structural equation modeling (ESEM), have recently been proposed as an overarching statistical framework making it possible to combine EFA, CFA, and 
SEM components in a single analytical model (Asparouhov \& Muthen, 2009; Marsh, Morin, Parker, \& Kaur, 2014; Morin, Marsh, \& Nagengast, 2013). The demonstrated benefits of ESEM over CFA lies in the ability to freely estimate all cross-loadings between items and non-target factors. Indeed, the statistical literature has recently shown ESEM to result in more accurate estimates of the factor correlations when cross-loadings (even as small as .100: Marsh, Lüdtke, Nagengast, Morin, \& Von Davier, 2013) are present in the population model, and to remain unbiased in the absence of crossloadings (for a review, see Asparouhov, Muthén, \& Morin, 2015). For these reasons, Guay, Morin, Litalien, Valois, and Vallerand (2015) suggested that ESEM might provide a more accurate and definitive test of the factor correlations identified in common measures of motivation, and thus provide a more exact assessment of whether these correlations follow the expected simplex pattern. Guay et al. (2015) addressed this question among two independent samples of college and high school student who completed the Academic Motivation Scale (Vallerand et al., 1992). As predicted, the ESEM solution resulted in a higher level of discriminant validity between the behavioral regulations factors, as well as in a closer, albeit imperfect, approximation of the expected simplex pattern relative to CFA. These authors also replicated these results among two independent samples of graduate students (Litalien, Guay, \& Morin, 2015).

Taken together, these factor analytic approaches strongly support the need for a multidimensional conceptualization of SDT motivation and explicitly disconfirm simplified one-dimensional measurement models. However, these approaches have demonstrated support for a multidimensional representation of motivation as encompassing multiple types of behavioral regulations, themselves displaying a pattern of correlation matching the expected continuum structure of self-determination. These results indicate a more nuanced interpretation implying the presence of distinct regulation types that follow a general ordering.

\section{Rasch Analysis}

Rather than focusing on factor analysis, correlations, or regressions among conceptually distinct behavioral regulations, Chemolli and Gagné (2014) conducted an even more stringent test of the onedimensional continuum structure of motivation by testing whether a single dimension could be assumed to provide an adequate representation of responses to multidimensional motivation measures. These authors applied Rasch analysis (Rasch, 1960) to the Academic Motivation Scale (Vallerand et al., 1992) and the Multidimensional Work Motivation Scale (Gagné et al., 2015). Rasch analysis is an item response theory model (Lord, 1980) in which all item responses are specified to fall along a single dimension (in this case, global self-determination). Items that fall further from this line are therefore measuring additional factors beyond the main Rasch dimension, and thereby indicate multidimensionality. This highly specific test failed to support the hypothesis that a single-dimension was sufficient to represent motivation as assessed in either of these two measures. Despite some notable criticism (Sheldon, Osin, Gordeeva, Suchkov, \& Sychev, 2017) ${ }^{1}$, this study once again provides evidence supporting the necessity of a multidimensional representation of human motivation.

\section{Multidimensional Scaling}

Multidimensional scaling is a predominantly exploratory alternative to factor analysis that depicts patterns and distances among variables in a multi-dimensional space (Jaworska \& ChupetlovskaAnastasova, 2009). This technique has been used to statistically compare single- and multidimensional representations of motivation and to produce estimates of how much of the structure each dimension is able to explain. Multidimensional scaling graphically represents the relative position of each regulation factor, which allows for an interpretation of how evenly these factors are spaced. If behavioral regulations follow a continuum, then a one-dimensional representation should provide satisfactory fit of the data and lead to a graphical representation showing regulations in a linear pattern representing increasing levels of self-determination. Alternatively, the presence of additional dimensions would indicate that a more complex multi-faceted representation is warranted. 
Howard et al., (2017) present the most comprehensive application of multidimensional scaling to date, applying it meta-analytically to over 480 samples. This analysis found that a single dimension was capable of explaining most of the structure of behavioral regulations. Additionally, the resulting graphical representation strongly resembled the proposed continuum structure with quite evenly spaced regulations located in their expected place on the hypothetical continuum of self-determination, with the exception of integrated regulation. However, a model incorporating a second dimension improved model fit, even if the improvement remained small. These results indicate that while a continuum structure reflecting the degree of self-determination may well be a core component of human motivation, there was still value in considering the unique characteristics of individual regulations over and above this global component. Another recent study by Sheldon et al. (2017) supported these conclusions in a combined sample of 1,547 participants who completed their newly developed Universal Perceived Locus of Causality Scale. Across four samples, between $38-71 \%$ of variance in behavioral regulations was explained by the self-determination continuum (i.e. the first dimension). However, a two-dimensional model fitted the data equally well. When presented graphically, this two-dimensional solution demonstrated a clear semi-circular shape, indicating that a more complex multidimensional interpretation might be necessary. Results from Howard et al. (2017) found a similar semi-circular representation. Sheldon et al. interpreted the second dimension to possibly represent the degree of effort of self-control required within each type of regulation: Amotivation and intrinsic motivation would score low on effort, while introjection would score the highest. This hypothesis could be verified in future empirical research. Together these results generally support the continuum hypothesis, but also indicate that a more complex interpretation may be required.

\section{A General Factor Representing the Continuum and Co-Existing Specific Factors}

Most approaches described above have either implicitly or explicitly opposed a multidimensional representation of the behavioral regulations against a one-dimensional representation of the continuum structure. As such, none of these tests directly considered integrating these two aspects of SDT's conceptualization of motivation in which human motivation encompasses categorically distinct behavioral regulations, themselves organized along a single continuum of self-determination. Howard et al. (2018) recently suggested that bifactor-ESEM approach (Morin, Arens, \& Marsh, 2016) might provide a way to reconcile these two perspectives and provide a way to integrate Guay et al. (2015) and Chemolli and Gagné (2014) approaches in a single model. Bifactor modeling relies on a global factor (the G-factor) to capture all variance that is shared among all items (as in the Rasch approach), but also allows each specific subscale to be estimated over and above the variance explained by the Gfactor, resulting in a series of subscale-specific factors (the S-factors) simultaneously estimated via an ESEM approach allowing for cross-loadings. In other words, items load onto both their respective subscales as in a standard ESEM solution (the S-factors), while also loading onto a G-factor (Reise, 2012) providing a direct representation of SDT continuum. Loadings on the G-factor should follow the continuum hypothesis, with items representing more self-determined forms of motivation (e.g., intrinsic motivation) showing strong positive loadings and items representing less self-determined motivation forms (e.g., external regulation) showing weaker loadings.

An early study by Gunnell and Gaudreau (2015) applied bifactor-CFA to the responses provided to the Behavioral Regulation in Exercise Questionnaire (BREQ-2) by a small sample of 186 university students. Their results supported the idea that a G-factor representing global motivation could coexists with $\mathrm{S}$-factors representing the behavioral regulation scales, with all factors defined by satisfactory factor loadings. However, the observed pattern of factor loadings on the G-factor did not follow the SDT's continuum hypothesis, but rather seemed to simply reflect participants' global level of motivation across all types of behavioral regulations. Nonetheless, Gunnell and Gaudreau (2015) found this general factor to be a strong predictor of physical activity and goal progress. However, they 
also found significant relations between identified and intrinsic regulations and physical activity, indicating the added value of some regulation subscales, beyond that of the general motivation factor.

In a more recent study of a large sample of 1124 Canadian employees who completed the Multidimensional Work Motivation Scale, and applying the more advanced bifactor-ESEM framework, Howard et al. (2018) found strong evidence for the co-existence of a G-factor matching the self-determination continuum, and meaningfully defined S-factors. Indeed, factor loadings were high and positive for intrinsic and identified regulations, moderately positive for introjected regulation, weak and non-significant for external regulation, and high and negative for amotivation. These factor loadings indicate that the self-determination continuum is bipolar in nature, and theoretically bounded by strong positive (+1) to strong negative (-1) factor loadings (Tay \& Jebb, 2018). In addition, Howard et al. showed that while the continuum G-factor was significantly associated with most of the covariates considered in their study (affective commitment and the satisfaction of employees' need for autonomy, competence, and relatedness), the S-factors were able to explain a significant level of additional variance (i.e., not already explained by the G-factor) in most of these outcomes in a manner that was consistent with SDT. Litalien, Morin, Gagné, Vallerand, Losier, and Ryan (2017) were able to replicate Howard et al.'s (2018) results in two independent samples of undergraduate and graduate university students who completed the Academic Motivation Scale. These authors also supported the idea that a G-factor corresponding to the SDT continuum hypothesis co-existed with S-factors reflecting the unique characteristics of behavioral regulations. They likewise showed that the G-factor presented significant positive associations with students' levels of vitality and satisfaction with their studies, and negative associations with their levels of ill-being and dropout intentions. Finally, they also showed that the $\mathrm{S}$-factors were able to explain additional variance in the covariates over and above that already explained by the G-factor.

These results present an integrative and nuanced view of motivation in which it becomes possible to empirically distinguish the global degree of self-determined motivation (corresponding to the SDT continuum) from the specific characteristics associated with each behavioral regulation. When considering these S-factors, it is important to keep in mind that their interpretation differs from that of typical first-order factor. More precisely, whereas scores on a typical first-order intrinsic motivation factor reflects the desire to pursue an activity for the pleasure and stimulation that it provides, the intrinsic motivation S-factor derived through B-ESEM rather reflects what remains in intrinsic motivation once participants' global levels of self-determination are taken into account. More specifically, these S-factors can be taken to reflect imbalance in participants' specific levels of behavioral regulations over and above their global levels of self-determination. These S-factors may thus reflect pure pleasure for intrinsic motivation, a match with one's personal values for identified regulation, guilt or shame for introjected regulation, pressure for external regulation, and a lack of intentionality for amotivation. Importantly, simultaneously considering the role of these various global and specific components in prediction provides additional information. In this instance, the results were found to favor the importance of a self-determination continuum, but also highlighted the added importance of the specific regulation subscales.

\section{Motivation Profiles}

A final approach to examine the continuum hypothesis of motivation, which allows subscale specificity to co-exist alongside the underlying continuum structure, is provided by person-centered analyses. This approach aims to identify subgroups of participants (i.e., profiles) presenting different configurations of the various behavioral regulations. Through examination of the distribution of the behavioral regulations among the most commonly endorsed profiles, inferences can be drawn about the presence of an underlying continuum of self-determination. More precisely, the continuum hypothesis would be supported by the observation of profiles represented by a single dominant regulation, with some endorsement of adjacent behavioral regulations, and decreasing levels of 
endorsement of more distal regulations. Such a profile would display a smooth unimodal curve. Alternatively, profiles characterized by matching levels of endorsement of non-adjacent behavioral regulations would argue against the continuum hypothesis. For example, according to the continuum hypothesis, profiles characterized by high levels of introjected and external regulations, lower levels of identified regulation, and even lower levels of intrinsic motivation should be far more common than profiles characterized by high level of both intrinsic motivation and external regulation.

The research literature conducted using this approach is quite extensive, though not generally designed to test the continuum hypothesis. Still, published person-centered research reveals that most studies, regardless of domain, tend to produce profiles characterized by unimodal curves (Bechter, Dimmock, Howard, Whipp, \& Jackson, 2018; Graves, Cullen, Lester, Ruderman, \& Gentry, 2015; Howard, Gagné, Morin, \& Van den Broeck, 2016; Liu, Wang, Tan, Koh, \& Ee 2009; Matsumoto, \& Takenaka, 2003; Moreno-Murcia, Gimeno, Hernández, Pedreño, \& Marín, 2013; Ntoumanis, 2002; Sheldon et al., 2017). However, a number of additional studies also found profiles that did not fully conform to this expected pattern (Boiche, Sarrazin, Grouzet, Pelletier, \& Chanal, 2008; McNeill \& Wang, 2005; Moran, Diefendorff, Kim, \& Liu, 2012; in de Wal, den Brok, Hooijer, Martens, \& van den Beemt, 2014; Ullrich-French, Cox, 2009; Yli-Piipari, Watt, Jaakkola, Liukkonen, \& Nurmi, 2009). Interestingly, many of the divergent profiles seemed to involve very small sample sizes, suggesting that they may either reflect random sampling variation or unidentified multivariate outliers.

A recent study specifically designed to test the continuum hypothesis in the physical education context with a sample of 3220 students is worth considering. In this study, Wang, Morin, Ryan, and Liu (2016) contrasted two alternative representations of the behavioral regulations, one based on the a priori dimensions of external regulation, introjected regulation, identified regulation, and intrinsic motivation with one based on the two higher-order dimensions of autonomous and controlled motivation. Interestingly, the profiles identified using the four a priori regulations subscales more closely followed the continuum hypothesis than the profiles identified using the higher-order dimensions. Most importantly their results also demonstrated that deviations from the continuum observed in the second (higher-order) scenario were due to the loss of information related to the merging of substantively meaningful sub-dimensions into higher-order dimensions. As such, this study supports both the continuum hypothesis, and the need to use measures of the regulations separately.

In summary, research in this area generally supports the notion of a continuum structure among the regulations, as profiles typically tend to follow unimodal curves. However, enough violations of this curve assumption have been noted to suggest that motivation cannot be entirely subsumed by a single one-dimensional continuum. It is worth noting that in these profile analyses, the effects associated with either the continuum or individual regulations are conflated and very difficult to parse. As such, future research in this area may require reliance on combinations of bifactor modeling and latent profile analysis in order to explicitly model the continuum while simultaneously including regulation subscales (Morin, Boudrias, et al, 2016, 2017).

\section{A Proposition: The Semi-Radex Structure}

The aim of this review was to explore whether motivation, as conceptualized according to SDT (Deci \& Ryan, 1985; Ryan \& Deci, 2017), follows a one-dimensional continuum structure, whether it encompasses several categorically distinguishable concepts, or both. Evidence from the range of tests described above does not present decisive evidence towards either the hypothesis that motivation is only a continuum, or that it encompasses multiple categorically distinct regulation types. Instead, results indicate that motivation varies both in degree (the overall amount of self-determination) and in kind (the types of regulations) simultaneously. While behavioral regulations seem to be ordered predictably in a simplex-like manner representing the degree of self-determination as observed by Ryan and Connell (1989), this ordering does not represent a true simplex as each individual regulation remains a separate construct with its own unique characteristics upon which individuals are free to 
score either high or low irrespective of their position on the self-determination continuum.

This raises an issue central to this review: Current conceptualizations of motivation do not account for this complex structure well, resulting in theoretical confusion and inconsistent use of motivation constructs. In other words, a more integrative and complex concept than a continuum or simplex is required to fully describe motivation in SDT. Fortunately, further examination of Guttman's Radex theory (1954) identifies several alternative conceptual models which can help describe multidimensional constructs.

Guttman's Radex theory notes that sets of variables can differ from one another in kind, in degree, or both, leading to three possible structures: a simplex (i.e., variables differ from one another in degree only), a circumplex (i.e., variables differ from one another in kind only), and a radex (i.e., variables differ from one another in kind and in degree). As noted by Ryan and Connell (1989), a simplex structure refers to a set of variables of the same kind which are arranged according to a simple order of degree. Variables following a simplex structure measure the same underlying construct, but to a greater or lesser degree, as illustrated in Figure 2. For example, a mathematics achievement test may include a variety of subtests organized according to their level of complexity so that numerical abilities required to master the least complex subtests (e.g., addition) would also be required to master more complex tests (e.g., multiplication), which themselves will tap into abilities useful to solve even more complex numerical problems (e.g., solving equations). As such, the subtests included in this test of mathematics achievement would form a simplex pattern as they all measure a single construct (i.e., mathematics ability) through items differing in their degree of complexity. The notion of "complexity" is often used when referring to Guttman' (1954) original writings and was indeed used by Guttman himself. However, the key aspect of Guttman' (1954) theory remains the presence of an ordering in the degree to which some characteristic is expressed. When focusing on intelligence or achievement, as in Guttman's (1954) work, then this ordering is expressed in terms of complexity. When focusing on motivation, this ordering then becomes one of self-determination (Ryan \& Connell, 1989).

Before moving further, it is important to note that Guttman's (1954) simplex is a single dimensional space, and thus assumes that an individual can only reside at one point along such a structure at any one time (e.g., a student cannot simultaneously master calculus and yet fail to understand the basic principles of multiplication). In contrast, one of the fundamental principles of SDT states that motivation is not a developmental or stage theory requiring direct progression through the regulations (Gagné \& Deci, 2005). Instead SDT suggests that individuals can change between regulations freely dependent upon the degree of need satisfaction and subsequent internalization. Moreover, it has been acknowledged that people usually hold more than one reason (regulation) for engaging in specific behaviors at any one time (Chemolli \& Gagné, 2014), as evidenced by latent profile analyses (Graves et al., 2015; Howard et al., 2016; Moran, Diefendorff, Kim, \& Liu, 2012). In other words, while the ordering of the subscales was always expected to follow a continuum structure according to SDT, individuals were never expected to be located at a single point along this selfdetermination continuum and this continuum structure was never expected by SDT to subsume its own individuals' behavioral regulations. As such, Guttman's (1954) simplex was never a true representation of SDT's theoretical underpinnings. Though motivational regulations do differ in their degree of self-determination, there are other meaningful differences between these regulations that are not captured by this continuum, indicating that the collection of subscales together requires a more complex structure than a simplex.

An alternative model proposed by Guttman (1954) is the circumplex, which refers to variables differing from one another in kind only, and not ordered in terms of degree. In the intervening years, this definition has been reformulated to describe any two continuous variables depicted graphically as intersecting axes in a two-dimensional space. For example, for any two given variables, this structure would be represented according to a biaxial system depicting the first variable along an $\mathrm{X}$-axis, and 
the other along a Y-axis. Circumplex structures are widely used in psychology, for example to represent affect (e.g., Russell, 1980). For instance, Figure 3 presents a circumplex model of affect, organized in terms of pleasantness and intensity, along which different affective states can be located. While each of these dimensions can be described individually as a continuum, when these two continua are combined to form a two-dimensional construct, they form a circumplex along which different characteristics can be located. More precisely, the variables accounted for by the circumplex presented in Figure 3 are pleasantness and intensity. These two variables each form their own continuum and are not expected to be related to one another. The resulting circumplex can then be used to classify affective states based on these two dimensions.

When transposed to motivation, a similar circumplex could be proposed, for instance, to be organized along one first dimension reflecting global self-determination, and a second dimension reflecting the effort invested, as suggested by Sheldon et al. (2017). Using the resulting circumplex, it would then become possible to locate participants' motivation for distinct activities along these two dimensions, assuming that a direct measure of effort expenditure was also available. However, adopting this approach would require the behavioral regulations to be entirely subsumed into these two dimensions, thereby requiring no unique or meaningful characteristics to be associated with the behavioral regulations. However, the large body of factor analytic and latent profile analytic research evidence has not supported this proposition, instead providing support for more than two dimensions underlying motivation, with dimensions representing each type of regulation themselves organized along a single overarching continuum.

Moreover, if we move beyond this circular representation of the circumplex, to go back to Guttman (1954) theoretical definition of constructs differing only in kind, this approach would be consistent with a representation of human motivation according to which each type of behavioral regulation differs in kind from the other, without being ordered along a single overarching continuum. This view does not match the results from previous research any more than the simplex does.

Finally, Guttman (1954) defines a radex as a structure in which multiple variables differ from one another both in kind and in degree. Figure 4 provides an example radex in which the subscales are organized among themselves in kind, while also differing in degree. Contrary to a circumplex, a radex makes most sense when an ordering is specified among the different types of variables. Thus, in a radex, bordering variables are assumed to be more closely related than non-bordering variables, allowing theoretical distances between concepts to be directly interpretable. In other words, a radex can incorporate any number of variables differing in kind (e.g. regulation types) and order them according to a central underlying principle (e.g. self-determination). To be as clear as possible, in the circumplex illustrated in Figure 3, the central axes represent the two dimensions, differing in kind, forming the conceptual structure. In contrast, no such axes are present in Figure 4. In Figure 4, four variables (subscales A, B, C, and D), differing in kind from one another, are depicted as sectors from the global conceptual model. Furthermore, these variables are expected to be organized along a central continuum, following the outline of the circle, and thus going from subscale $\mathrm{A}$, to subscale $\mathrm{B}$, to subscale $\mathrm{C}$, to subscale $\mathrm{D}$, and then back to subscale $\mathrm{A}$. In this radex representation, it then becomes possible to locate participants' motivation for distinct activities along each of the dimensions A-B-C$\mathrm{D}$, as well as around the circle itself depending on the relative strength of each dimension.

This radex structure neatly describes the complex multidimensional yet predictably ordered nature of motivation specified by SDT, as first pointed out by Chemolli and Gagné (2014). However, a true radex forms a complete circle where each segment is positioned as theoretically close to its neighboring concepts, and where a binary opposite directly opposes each concept in the circle. This same structure does not describe the motivational regulations proposed by SDT. For example, introjected regulation is closely associated with both external and identified regulations but does not represent a direct opposite to either intrinsic motivation or amotivation. Indeed, no direct binary 
opposite is proposed by SDT for any of the behavioral regulations. Likewise, intrinsic motivation and amotivation are highly divergent concepts and should not be adjacent factors in a radex structure but should rather located at each extreme. Still, they do remain conceptually different, as amotivation does not simply reflect the lack of "pleasure", but rather reflects a complete lack of intentionality.

Therefore, we argue that the best fitting representation of motivation would be a semi-radex, as illustrated in Figure 5. A semi-radex conceptualization is supported by the entirety of reviewed evidence including the predictable ordering noticed in correlation tables, factor analyses finding distinct regulations types, Rasch analysis suggesting that a single dimension in not sufficient, bifactor modeling which identifies both self-determination and regulation-specific factors, multidimensional scaling revealing a strong but not sufficient central dimension, and the commonly observed shapes of motivation profiles. It is worth noting that traditional analyses to test for a radex structure (e.g. smallest space analysis) fall under the category now known as multidimensional scaling. As reviewed above, recent evidence of this kind supports the presence of a radex structure within regulation types and even items conforming to a semi-circle shape when depicted graphically. In sum, the evidence presented in this review suggests that a semi-radex conceptualization is best suited to the theoretical examination of motivation and provides a way to describe the behavioral regulations as being both distinct, yet still predictably ordered, as displayed in Figure 5. More precisely, in this semi-radex representation, it becomes possible to locate participants' motivation for distinct activities separately on each type of behavioral regulation, as well as around the semi-circle itself depending on the relative strength of each dimension.

\section{Theoretical, Practical, and Methodological Implications}

The major theoretical implication to be drawn from this review is that the continuum of selfdetermination and distinctiveness of the individual regulation types can, and should, be integrated into a single semi-radex conceptualization. This is important for practical reasons as both the degree of self-determination as well as unique regulation characteristics have been associated with important outcomes across life domains. For example, Litalien et al (2017) found that both the general factor representing the degree of self-determination and the unique characteristics associated with specific regulations were significant predictors of outcomes. Specifically, they found that both the degree of self-determination and specific regulation subscales significantly predicted vitality, ill-being, dropout intention, and satisfaction, and that while the degree of self-determination did not predict academic achievement, specific regulation subscales did. Howard et al (2018) further demonstrate this trend in finding that while the general self-determination factor was unrelated to continuance commitment to the organization, several of the regulation subscales were significantly related to this outcome variable. Additionally, both the global degree of self-determination and the specific regulations were important predictors of affective commitment to the organization. These conclusions have been replicated consistently over multiple domains via person-centered analyses (Bechter, et al., 2018; Howard et al., 2016; Wang et al., 2016). These examples all demonstrate the importance of acknowledging and modeling the full complexity of motivation with bifactor studies demonstrating this specifically in variable-centered approaches whereas profile studies show this from a holistic person-centered perspective.

The proposed semi-radex structure of human motivation resolves an ongoing debate in the scientific literature concerning the nature of motivation, hopefully uniting competing views (Chemolli \& Gagne, 2014; Sheldon et al., 2017; Howard et al., 2017) by specifying an evidence-based solution that sufficiently and concisely describes the nature of motivation specified within SDT. This unity brings notable benefits to SDT researchers more broadly. The semi-radex structure explicitly identifies and explains the two key components of the SDT-based conceptualization of motivation (the continuum and regulation types). As a result, this conceptualization will allow for clearer and more specific theorizing and hypothesis testing regarding which key elements are at play. This is opposed to 
current research practice which almost unanimously examines one without the other, leading to confounded or limited conclusions.

This theoretical refinement and integration are essential steps in the future development of SDT and, in addition to clearly specifying the key elements of motivation, will allow SDT researchers to speak the same language, thereby increasing the ability to integrate research findings in the future. Without such a unifying conceptualization, SDT research will continue to develop hypotheses based on either one or the other motivational aspect and use research methods and operationalizations that measure only one aspect (e.g. subscale scores, RAI, autonomous/controlled dichotomizations) which subsequently produces results that cannot be compared and integrated into a comprehensive corpus of knowledge. For example, a study examining the role of motivation in well-being using a latent variable to represent autonomous motivation will include everything that is common between identified and intrinsic motivation into the construct and will exclude (as residuals) elements that are unique to identified regulation (i.e., meaning) and intrinsic motivation (enjoyment). In contrast, a study using a bifactor model to represent self-determination, meaning, and enjoyment as separate latent factors, would likely provide more information about aspects of motivation important to wellbeing. This example highlights how the semi-radex structure will help prevent these confounded interpretations by encouraging more holistic studies that account for both key elements, potentially even distinguishing and measuring each of them within the study.

How then do we recommend operationalizing motivation when conducting research? Multidimensional operationalizations of the motivation construct through the specification of regulation subscales using CFA, ESEM, or multidimensional scaling would likely capture much of the information inherent within a semi-radex structure, though they fail to adequately disentangle the effects (i.e. continuum vs. regulations). On the other hand, composite measures of motivation, such as the relative autonomy index (RAI: Grolnick \& Ryan, 1989) may address the underlying continuum more specifically, though neglect to account for effects stemming directly from the regulations.

An alternate way to operationalize motivation which avoids many of the limitations of the previously mentioned methods, is through the estimation of bifactor models. The bifactor-ESEM framework provides a direct way of simultaneously assessing the presence of conceptually differentiated behavioral regulations co-existing with an overarching continuum of self-determination. The application of the bifactor-ESEM framework provides a way to simultaneously estimates participants' location on the underlying self-determination continuum estimated from their responses obtained across all motivation items (i.e., the G-factor), together with their specific levels on each of the specific types of behavioral regulation left unexplained by this G-factor (i.e., the S-factors). As such, this approach provides a way to simultaneously account for both of the core aspects of SDT representation of human motivation: The continuum (i.e., the global self-determination component) and specificity (i.e., the unique quality of each type of regulation). The first of those components, the G-factor, should be directly estimated from all motivation items included in the motivation measure under consideration. Loadings on this global factor should match the a priori continuum structure, with loadings ranging from 1 to -1 (i.e., strong and positive for intrinsic and identified regulations, moderately positive for introjected regulation, weak and non-significant for external regulation, and high and negative for amotivation; Howard et al., 2018; Litalien et al., 2017).

In addition, one specific factor should be incorporated for each type of self-regulation included in the instruments (e.g., five S-factors for intrinsic, identified, introjected, external, and amotivation). As with more traditional factor analytic approaches, the number of modeled S-factors will vary depending on the number of subscales included in the measure under consideration. These factors should be defined from their a priori items (through target rotation; Marsh et al., 2014), and orthogonal in order to achieve a proper disaggregation of the global versus specific sources of variance included in the measure (Morin, Myers, \& Lee, 2020). As noted above, these S-factors will then come to reflect 
imbalance in participants' specific levels of behavioral regulations over and above their global levels of self-determination.

The bifactor-ESEM approach makes it not only possible to represent both core components of motivation within SDT, but also to incorporate them into further analyses (i.e., prediction, trajectories, profiles, etc.) designed to advance our understanding of human motivation. For example, one could expect that the perceived meaningfulness of an action (i.e., the specific characteristic of identified regulation) could be more important in predicting certain behaviors (e.g. voting; Losier \& Koestner, 1999), whereas other outcomes may depend entirely on the global degree of self-determination.

Interested readers are referred to Morin, Arens and Marsh (2016) and Morin, Boudrias et al. (2017) for user-friendly introduction to this new analytic framework, to Morin, Myers, and Lee (2020) for a more conceptual introduction, and to Litalien et al. (2017) and Howard et al. (2018) for applications to motivation.

The application of person-centered approaches studying motivation profiles is also very promising. This approach avoids issues of multicollinearity as well as the statistical concerns of composite measures and provides a holistic and comprehensive perspective of an individual's motivation. Finally, it would be interesting to examine motivation profiles estimated based on preliminary bifactor-ESEM measurement models (Morin, Boudrias, et al., 2016, 2017). This approach would directly identify motivation profiles differing from one another in terms of differing types of motivation and overall degree of self-determination simultaneously.

\section{Limitations and Future Research}

A key direction for future research stemming from this review revolves around examination of added contribution concerning both the degree of self-determination and the regulation types. Stated otherwise, what specific roles do these factors play in predicting outcomes? Is one more important, or does it vary depending on the outcome or even contextual factors? Do these elements interact? What predicts each of these factors? The proposed conceptualization encourages these types of questions, and contemporary methods (e.g. bifactor models \& profile analysis) now allow them to be tested.

Additionally, future research might benefit from deeper analysis of the items used to measure motivation. For example, item response theory approaches or computer-adaptive testing might improve measurement by ensuring items are functioning optimally and are able to capture both very high and very low levels of a regulation (Tay \& Jebb, 2018). While current items are not designed to capture difficulty, this remains a potential area of further development.

Another notable question still to be sufficiently addressed is the role of amotivation within the SDT conceptualization of motivation. Amotivation is commonly measured in contemporary SDT scales and operationalized as a state of ambivalence (e.g. "I don't know why I'm doing this job, it's pointless" MWMS, Gagné et al., 2015). However, some note that a variable measuring a lack of motivation or unwillingness to exert effort is not comparable to the other regulation types and becomes particularly problematic when forming composite scores such as the RAI. As such, it is somewhat ambiguous as to whether the continuum of self-determination is bipolar in nature (Tay \& Jebb, 2018) and therefore ranges from -1 to +1 (i.e. amotivation to intrinsic motivation), or whether it is unipolar, ranging from 0 to +1 with external regulation representing the lowest end of the continuum. Based upon past research, we argue for a bipolar continuum $(-1$ to +1$)$ as represented in Figure 5. However, if further theoretical research were to establish amotivation as a distinct construct removed from the continuum of self-determination, the semi-radex would remain a suitable solution regardless. Amotivation would simply be removed from the semi-radex, leaving the four remaining regulation types to fill out the structure.

Finally, the current state of research in this area is almost entirely cross-sectional and as such does not address issues of stability and change in individual's motivation towards a behavior or context over time. Specifically, this issue would be essential in informing the debate about whether people 
truly experience multiple regulations at any given time, or whether people cycle through individual regulations in sequence over time. For example, suppose an individual reports strong feelings of introjected regulation at work, as well as lower levels of external and identified regulations. When this individual decides to resume work after a self-imposed break, this decision could be driven by a single instance of pure introjected regulation or by a more complex weighing up of multiple regulatory processes occurring simultaneously. If decisions to enact behaviors such as this are in fact the result of a single impulse of one type of regulation at a specific point in time, then this would be entirely in line with the classical simplex-like continuum hypothesis. Other regulating factors may be experienced before or after the decision, but the instigating motivator could be limited to single type of regulation, and information that would be missed in research focusing on "generic" motivational orientations. In contrast, if decisions to enact behaviors are anchored in more complex combinations of regulations, then the semi-radex representation would be more appropriate. Unfortunately, the vast majority of research on motivation is conducted at the domain level (Vallerand, 1997), focusing on motivation across an entire life domain, which may explain why individuals always report multiple regulations. For this reason, longitudinal research, as well as research relying on ecological momentary assessments methods providing a way to access immediate reasons for behavior, appear to be particularly promising directions for future research.

\section{Conclusion}

Motivation as defined by SDT, which has traditionally concurrently been described as unique elements and described using a continuum of self-determination, can now be integrated and described as a semi-radex. In this semi-radex, motivational regulations both retain unique properties while being ordered in a predictable manner. As well as resolving an ongoing debate concerning the simplex structure often cited in the literature, this semi-radex structure refines SDT theoretically by providing an integrative and nuanced conceptualization of motivation. As such, this will help foster more precise theorizing and testing of the two key elements of SDT alongside one another, and furthermore allow for easier integration of research findings.

\section{Footnotes}

${ }^{1}$ Advocates of the simplex approach (Sheldon et al., 2017) have argued that such one factor approaches are incompatible with a simplex representation based on statistical evidence showing that a simplex correlation matrix could best be reflected by two alternative two-factor models. We address this criticism as part of a webnote (https://osf.io/6kr25/?view only=019bb08dc8624d34affb5400ba85efe6) showing in fact that this same statistical evidence can be used to support the bifactor approach described later in this manuscript.

\section{Compliance with Ethical Standards:}

Funding: This study was funded by the Monash Business School, Monash University (grant number 1759764), awarded to the first author.

Ethical Approval: This article does not contain any studies with human participants performed by any of the authors. 


\section{References}

Asparouhov, T., \& Muthén, B. (2009). Exploratory structural equation modeling. Structural Equation Modeling, 16, 397-438.

Asparouhov, T., Muthén, B., \& Morin, A. J. (2015). Bayesian structural equation modeling with crossloadings and residual covariances. Journal of Management, 41, 1561-1577.

Bechter, B.E., Dimmock, J.A., Howard, J.L., Whipp, P.R., \& Jackson, B. (2018). Student motivation in high school physical education: A latent profile analysis approach. Journal of Sport and Exercise Psychology, 40(4), 206-216.

Boiche, J., Sarrazin, P., Grouzet, F., Pelletier, P., \& Chanal, J. (2008). Students' motivational profiles and achievement outcomes in physical education: A self-determination perspective. Journal of Educational Psychology, 100, 688-701.

Cerasoli, C., Nicklin, J., \& Ford, M. (2014). Intrinsic motivation and extrinsic incentives jointly predict performance: A 40 year meta-analysis. Psychological Bulletin, 140, 980-1008.

Chatzisarantis, N.L., Hagger, M.S., Biddle, S.J.H., Smith, B., \& Wang, J.C.K. (2003). A meta-analysis of perceived locus of causality in exercise, sport, and physical education contexts. Journal of Sport \& Exercise Psychology, 25, 284-306.

Chemolli, E., \& Gagné, M. (2014). Evidence against the continuum structure underlying motivation measures derived from self-determination theory. Psychological Assessment, 26, 575-585.

deCharms, R. (1968). Personal causation: The internal affective determinants of behavior. New York: Academic Press

Deci, E.L., \& Ryan, R.M. (1985). Intrinsic motivation and self-determination in human behavior. New York: Plenum.

Deci, E.L., \& Ryan, R.M. (2000). The" what" and" why" of goal pursuits: Human needs and the selfdetermination of behavior. Psychological Inquiry, 11, 227-268.

Deci, E.L., Koestner, R., \& Ryan, R.M. (1999). A meta-analytic review of experiments examining the effects of extrinsic rewards on intrinsic motivation. Psychological Bulletin, 125, 627-668.

Deci, E.L., Koestner, R., \& Ryan, R.M. (2001). Extrinsic rewards and intrinsic motivation in education: Reconsidered once again. Review of Educational Research, 71, 1-27.

Fernet, C., Senécal, C., Guay, F., Marsh, H., \& Dowson, M. (2008). The Work Tasks Motivation Scale for Teachers (WTMST). Journal of Career Assessment, 16, 256-279.

Fishbein, M., \& Ajzen, I. (1977). Belief, attitude, intention, and behavior: An introduction to theory and research.

Gagné, M., \& Deci, E.L. (2005). Self-determination theory and work motivation. Journal of Organizational Behavior, 26, 331-362.

Gagné, M., Forest, J., Vansteenkiste, M., Crevier-Braud, L., Van den Broeck, A., Aspeli, A.K., ... \& Halvari, H. (2015). The multidimensional work motivation scale: Validation evidence in seven languages and nine countries. European Journal of Work \& Organizational Psychology, 24, 178-196.

Graves, L.M., Cullen, K.L., Lester, H.F., Ruderman, M.N., \& Gentry, W.A. (2015). Managerial motivational profiles: Composition, antecedents, and consequences. Journal of Vocational Behavior, 87, 32-42.

Grolnick, W.S., \& Ryan, R.M. (1989). Parent styles associated with children's self-regulation and competence in school. Journal of Educational Psychology, 81, 143.

Guay, F., Morin, A.J., Litalien, D., Valois, P., \& Vallerand, R.J. (2015). Application of exploratory structural equation modeling to evaluate the academic motivation scale. The Journal of Experimental Education, 83, 51-82.

Guay, F., Vallerand, R.J., \& Blanchard, C. (2000). On the assessment of situational intrinsic and extrinsic motivation: The Situational Motivation Scale. Motivation \& Emotion, 24, 175-213.

Gunnell, K.E., \& Gaudreau, P. (2015). Testing a bi-factor model to disentangle general and specific 
motivation in self-determination theory. Personality \& Individual Differences, 81, 35-40.

Guttman, L. (1954). A new approach to factor analysis: the Radex. In Lazarsfeld, Paul. F. (ed.), Mathematical thinking in the social sciences. Glencoe, Ill: The Free Press.

Howard, J.L., Gagné, M., Bureau, J.S. (2017). Testing a continuum structure of self-determined motivation: A meta-analysis. Psychological Bulletin. 143, 1346.

Howard, J.L., Gagné, M., Morin, A.J.S., \& Forest, J. (2018). Using bifactor exploratory structural equation modeling to test for a continuum structure of motivation. Journal of Management, 44, 2638-2664.

Howard, J., Gagné, M., Morin, A.J.S., \& Van den Broeck, A. (2016). Motivation profiles at work: A self-determination theory approach. Journal of Vocational Behavior, 95, 74-89.

in de Wal, J.J., den Brok, P.J., Hooijer, J.G., Martens, R.L., \& van den Beemt, A. (2014). Teachers' engagement in professional learning. Learning \& Individual Differences, 36, 27-36.

Jaworska, N., \& Chupetlovska-Anastasova, A. (2009). A review of multidimensional scaling (MDS) and its utility in various psychological domains. Tutorials in Quantitative Methods for Psychology, 5, 1-10.

Koestner, R., Losier, G.F., Vallerand, R.J., \& Carducci, D. (1996). Identified and introjected forms of political internalization: extending self-determination theory. Journal of Personality \& Social Psychology, 70, 1025-1036.

Li, F. (1999). The exercise motivation scale: Its multifaceted structure and construct validity. Journal of Applied Sport Psychology, 11, 97-115.

Li, F., \& Harmer, P. (1996). Testing the simplex assumption underlying the Sport Motivation Scale: A structural equation modeling analysis. Research Quarterly for Exercise and Sport, 67, 396-405.

Litalien, D., Guay, F., \& Morin, A.J. (2015). Motivation for PhD studies: Scale development and validation. Learning \& Individual Differences, 41, 1-13.

Litalien, D., Morin, A.J.S., Gagné, M., Vallerand, R.J., Losier, G.F., Ryan. R.M. (2017). Evidence of a continuum structure of academic self-determination: A two-study test using a bifactor-ESEM representation of academic motivation. Contemporary Educational Psychology. 51, 67-82.

Liu, W., Wang, J., Tan, O., Koh, C., \& Ee, J. (2009). A self-determination approach to understanding students' motivation in project work. Learning \& Individual Differences, 19, 139-145.

Locke, E. A., \& Latham, G. P. (1990). A theory of goal setting \& task performance. Prentice-Hall, Inc.

Lonsdale, C., Hodge, K., \& Rose, E.A. (2008). The development of the Behavioral Regulation in Sport Questionnaire (BRSQ). Journal of Sport \& Exercise Psychology, 30, 323-335.

Lord, F.M. (1980). Applications of item response theory to practical testing problems. Mahwah, NJ: Routledge.

Losier, G.F., \& Koestner, R. (1999). Intrinsic versus identified regulation in distinct political campaigns: The consequences of following politics for pleasure versus personal meaningfulness. Personality and Social Psychology Bulletin, 25, 287-298.

Mallett, C., Kawabata, M., Newcombe, P., Otero-Forero, A., \& Jackson, S. (2007). Sport motivation scale-6: A revised six-factor sport motivation scale. Psychology of Sport \& Exercise, 8, 600-614.

Marsh, H.W., Lüdtke, O., Nagengast, B., Morin, A.J., \& Von Davier, M. (2013). Why item parcels are (almost) never appropriate: Two wrongs do not make a right - Camouflaging misspecification with item parcels in CFA models. Psychological Methods, 18, 257-284.

Marsh, H., Morin, A., Parker, P., \& Kaur, G. (2014). Exploratory structural equation modeling: An integration of the best features of exploratory and confirmatory factor analysis. Annual Review of Clinical Psychology, 10, 85-110.

Matsumoto, H., \& Takenaka, K. (2003). Motivational profiles and stages of exercise behaviour change. International Journal of Sports \& Health Science, 2, 89-96.

McClelland, D. C. (1987). Human Motivation. Cambridge Univ. Press, New York. 
McNeill, M., \& Wang, J. (2005). Psychological profiles of elite school sports players in Singapore, Psychology of Sport \& Exercise, 6, 117-128.

Moran, M.J.S., Diefendorff, J., Kim, T., \& Liu, Z. (2012). A profile approach to self-determination theory motivations at work. Journal of Vocational Behavior, 81, 354-363

Morin, A.J.S., Arens, A., \& Marsh, H. (2016). A bifactor exploratory structural equation modeling framework for the identification of distinct sources of construct-relevant psychometric multidimensionality. Structural Equation Modeling, 23, 116-139.

Morin, A.J.S., Boudrias, J.-S., Marsh, H.W., Madore, I., \& Desrumaux, P. (2016). Further reflections on disentangling shape and level effects in person-centered analyses: An illustration aimed at exploring the dimensionality of psychological health. Structural Equation Modeling, 23, 438-454.

Morin, A.J.S., Boudrias, J.-S., Marsh, H.W., McInerney, D.M., Dagenais-Desmarais, V., \& Madore, I. (2017). Complementary variable- and person-centered approaches to exploring the dimensionality of psychometric constructs: Application to psychological wellbeing at work. Journal of Business \& Psychology, 32, 395-419.

Morin, A.J.S., Marsh, H., \& Nagengast, B. (2013). Exploratory structural equation modeling: An introduction. In G. R. Hancock \& R. O. Mueller (Eds.), Structural Equation Modeling: A Second Course (2nd ed., pp. 395-436). Greenwich, CT: IAP.

Morin, A.J.S., Myers, N.D., \& Lee, S. (2020, In Press). Modern factor analytic techniques: Bifactor models, exploratory structural equation modeling (ESEM) and bifactor-ESEM. In G. Tenenbaum \& R.C. Eklund (Eds.), Handbook of Sport Psychology, 4th Edition. London, UK: Wiley

Moreno-Murcia, J., Gimeno, E., Hernández, E., Pedreño, N., \& Marín, M. (2013). Motivational profiles in physical education and their relation to the theory of planned behavior. Journal of Sports Science and Medicine, 12, 551-558.

Ng, J.Y., Ntoumanis, N., Thøgersen-Ntoumani, C., Deci, E.L., Ryan, R.M., Duda, J.L., \& Williams, G.C. (2012). Self-determination theory applied to health contexts a meta-analysis. Perspectives on Psychological Science, 7, 325-340.

Ntoumanis, N. (2002). Motivational clusters in a sample of British physical education classes. Psychology of Sport and Exercise, 3, 177-194

Pelletier, L.G., Fortier, M.S., Vallerand, R.J., Tuson, K.M., Briere, N.M., \& Blais, M.R. (1995). Toward a new measure of intrinsic motivation, extrinsic motivation, and amotivation in sports: The Sport Motivation Scale (SMS). Journal of Sport and Exercise Psychology, 17, 35-35.

Pelletier, L.G., Rocchi, M.A., Vallerand, R.J., Deci, E.L., \& Ryan, R.M. (2013). Validation of the revised sport motivation scale (SMS-II). Psychology of Sport and Exercise, 14, 329-341.

Rasch, G. (1960). Studies in mathematical psychology: I. Probabilistic models for some intelligence and attainment tests. Oxford, England: Nielsen \& Lydiche

Reeve, J. (2016). A grand theory of motivation: Why not? Motivation and Emotion, 40(1), 31-35.

Reise, S. (2012). The rediscovery of bifactor measurement models. Multivariate Behavioral Research, 47, 667-696.

Russell, J. A. (1980). A circumplex model of affect. Journal of Personality and Social Psychology, 39(6), 1161.

Ryan, R.M. (1995). Psychological needs and the facilitation of integrative processes. Journal of Personality, 63, 397-427.

Ryan, R.M., \& Connell, P. (1989). Perceived locus of causality and internalization. Journal of Personality \& Social Psychology, 57, 749-761.

Ryan, R.M., \& Deci, E.L. (2017). Self-determination theory: Basic psychological needs in motivation, development, and wellness. New York: Guilford.

Schwartz, S.H. (1992). Universals in the content and structure of values: Theory and empirical tests in 20 countries. In M. Zanna (Ed.), Advances in Experimental Social Psychology (Vol. 25, pp. 1- 
65). New York: Academic Press.

Sheldon, K.M., Osin, E.N., Gordeeva, T.O., Suchkov, D.D., \& Sychev, O.A. (2017). Evaluating the dimensionality of self-determination theory's relative autonomy continuum. Personality \& Social Psychology Bulletin, 43, 1215-1238.

Tay, L., \& Jebb, A.T. (2018). Establishing construct continua in construct validation: the process of continuum specification. Advances in Methods and Practices in Psychological Science, 1(3), 375388.

Ullrich-French, S., Cox, A. (2009). Using Cluster Analysis to Examine the Combinations of Motivation Regulations of Physical Education Students. Journal of Sport \& Exercise Psychology, 31, 358-379.

Vallerand, R.J. (1997). Toward a hierarchical model of intrinsic and extrinsic motivation. In Advances in experimental social psychology (Vol. 29, pp. 271-360). Academic Press.

Vallerand, R.J., Pelletier, L.G., Blais, M.R., Briere, N.M., Senecal, C., \& Vallieres, E.F. (1992). The academic motivation scale: A measure of intrinsic, extrinsic, and amotivation in education. Education and Psychological Measurement, 52, 1003-1017.

Van den Broeck, A., Carpini, J., \& Dieffendorf, J. (2019). Work motivation: Where do the different perspectives lead us? In R. Ryan (Ed.), The oxford handbook of human motivation (pp. 507-526). New York, NY: Oxford University Press.

Vroom, V. H. (1964). Work and motivation (Vol. 54). New York: Wiley.

Wang, J.C., Morin, A.J.S., Ryan, R.M., \& Liu, W.C. (2016). Students' Motivational Profiles in the Physical Education Context. Journal of Sport \& Exercise Psychology, 38(6), 612-630.

Yli-Piipari, S., Watt, A., Jaakkola, T., Liukkonen, J., \& Nurmi, J. (2009). Relationships between physical education students' motivational profiles, enjoyment, state anxiety, and self-reported physical activity, Journal of Sports Science and Medicine, 8, 327-336.

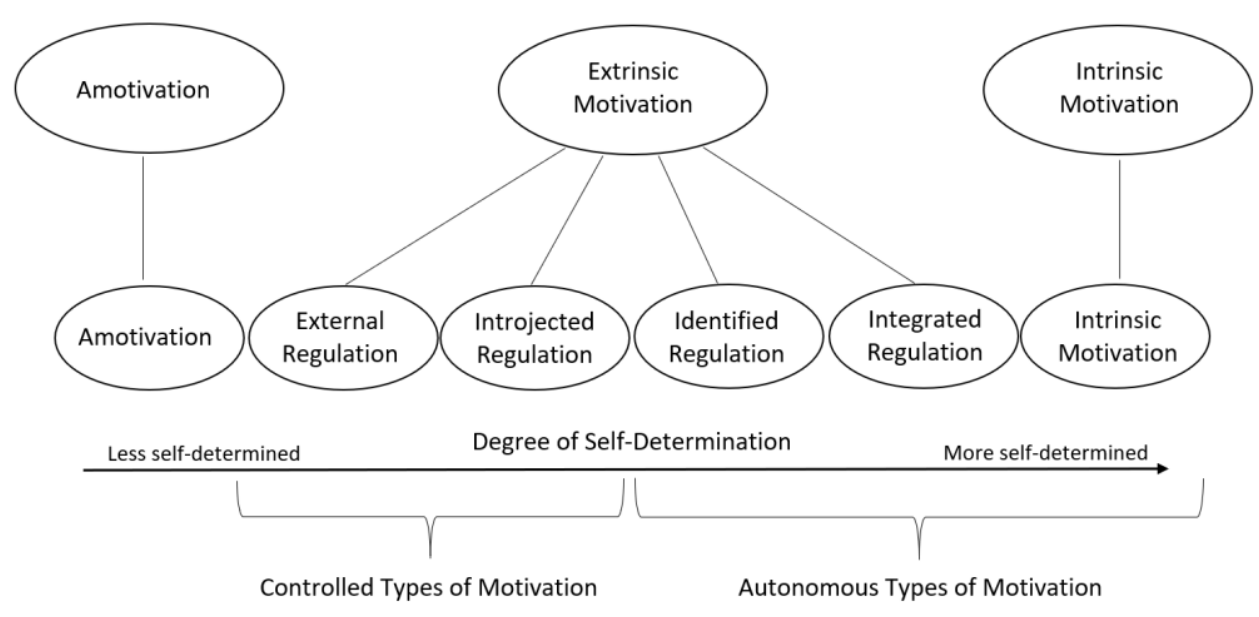

Figure 1. Representation of Motivation in Self-Determination Theory.

Note: Adapted from "Testing a Continuum Structure of Self-Determined Motivation: A MetaAnalysis," by J.L. Howard., M. Gagné., \& J.S. Bureau, 2017, Psychological Bulletin, 143(12), p. 1347. Copyright 2017 by American Psychological Association. 
Reviewing the Structure of Motivation 20

\begin{tabular}{|l|l|l|l|l|l|}
\hline$N_{1}$ & $N_{2}$ & $N_{3}$ & $N_{4}$ & $N_{5}$ & $N_{6}$ \\
\hline
\end{tabular}

\section{Figure 2. A Hypothetical Simplex}

Note: $\mathrm{N}_{1-6}$ represent subscales ordered in terms of the degree to which they reflect the underlying construct (increasing complexity for intelligence tests, increasing levels of self-determination for motivation tests). 


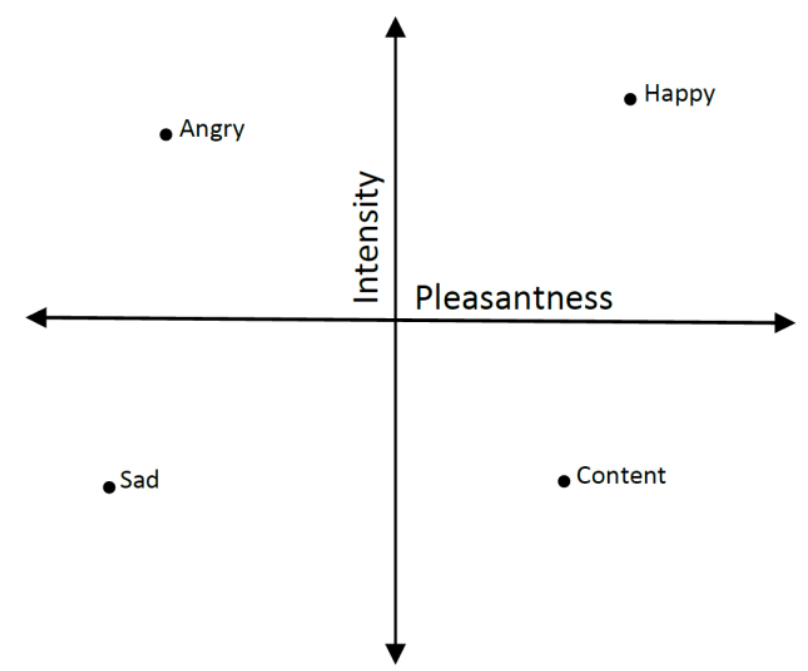

Figure 3. A Circumplex of Affect

Note: Adapted from “A circumplex model of affect," by Russell, J.A., 1980. Journal of Personality and Social Psychology, 39(6), p. 1168. Copyright 1980 by American Psychological Association.

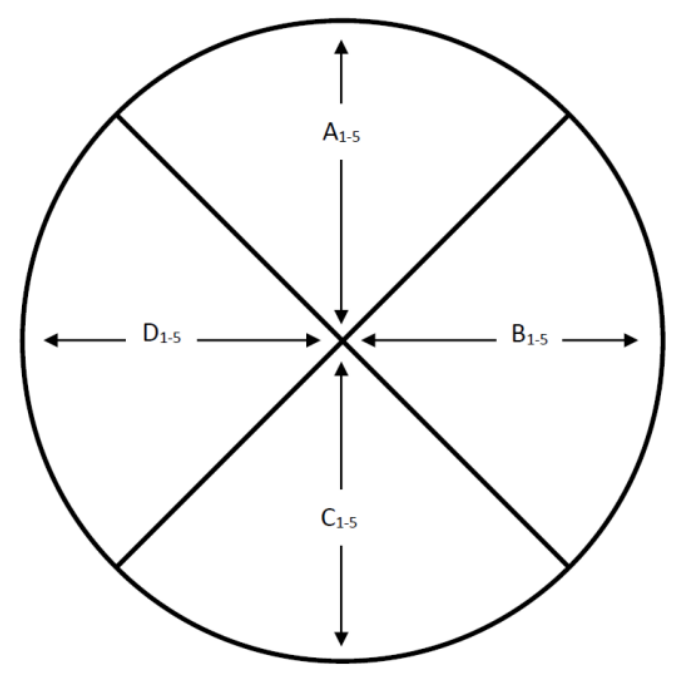

Figure 4. A Hypothetical Radex Structure

Note: Each subscale (A-D) differs from one another in both degree and kind. 


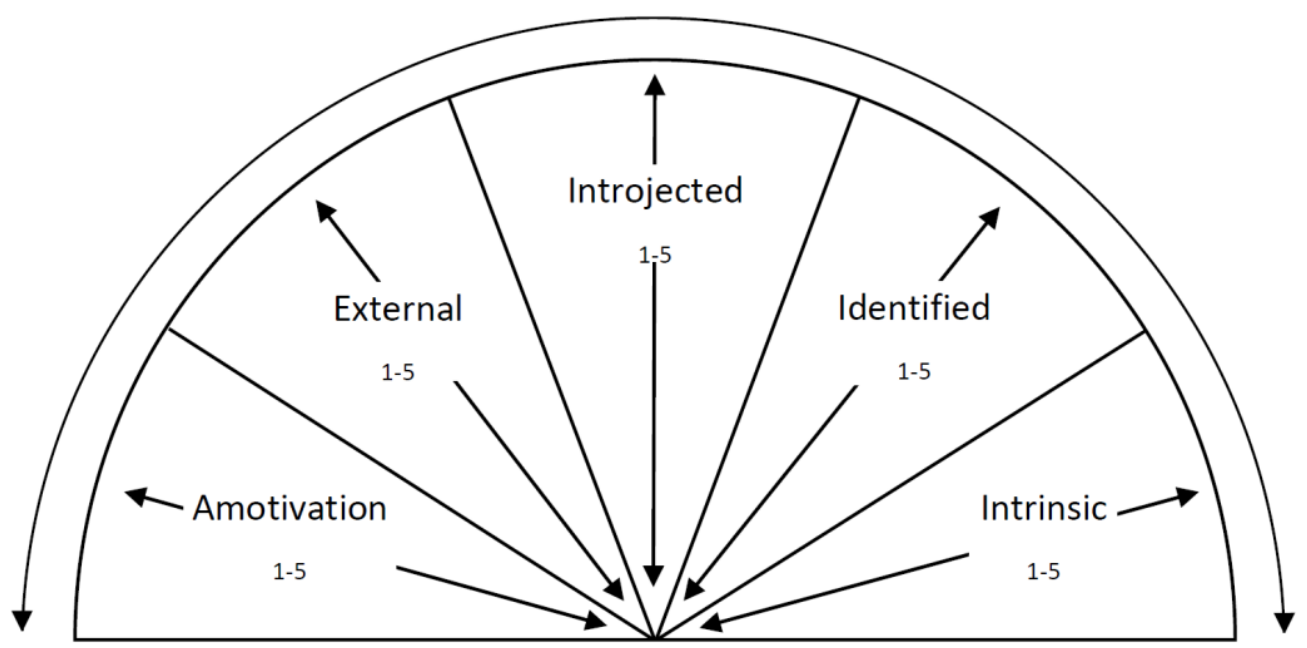

Figure 5. The Semi-Radex of Self-Determined Motivation

Note: ${ }_{1-5}$ indicates that in this example, each regulation type is measured by five items. The bidirectional arrow indicates the degree of self-determination and can range from low to high. However, individuals can vary further still based upon the degree to which they endorse each regulation. 